\title{
1-Quasiconformal Mappings and CR Mappings on Goursat Groups
}

\author{
Qing Yan Wu and Zun Wei Fu \\ Department of Mathematics, Linyi University, Linyi, Shandong 276005, China \\ Correspondence should be addressed to Zun Wei Fu; zwfu@mail.bnu.edu.cn
}

Received 27 February 2014; Accepted 2 April 2014; Published 24 April 2014

Academic Editor: Dachun Yang

Copyright (c) 2014 Q. Y. Wu and Z. W. Fu. This is an open access article distributed under the Creative Commons Attribution License, which permits unrestricted use, distribution, and reproduction in any medium, provided the original work is properly cited.

We show that 1-quasiconformal mappings on Goursat groups are CR or anti-CR mappings. This can reduce the determination of 1-quasiconformal mappings to the determination of CR automorphisms of CR manifolds, which is a fundamental problem in the theory of several complex variables.

\section{Introduction}

The well-known Liouville theorem tells us that 1-quasiconformal mappings on $\mathbb{R}^{n}$ are elements of group $\mathrm{SO}$ $(n+1,1)$. Quasiconformal mappings on the Heisenberg group were introduced by Mostow [1] in his study of rigidity theorem. Since then, quasiconformal mappings on nilpotent Lie group and on more general Carnot-Carathéodory spaces had drawn more and more concern. Korányi and Reimann [2] and Korányi and Reimann [3] showed that $C^{4}$ orientation preserving 1-quasiconformal mappings between domains of Heisenberg group $\mathbb{H}^{n}$ are CR or anti-CR mappings and are obtained by the restriction of $\mathrm{SU}(n+1,1)$ group actions (see also [4]). Cowling et al. [5] extended Liouville theorem to conformal mappings on $G / P$, where $G$ is a semisimple Lie group and $P$ is a minimal parabolic subgroup of $G$. Ottazzi and Warhurst $[6,7]$ obtained a Liouville type theorem for all Carnot groups which sated that 1-quasiconformal maps form finite dimensional Lie groups. Xie [8] investigated 1-quasiconformal mappings on Carnot groups with reducible first layer equipped with left-invariant sub-Riemannian Carnot metrics and showed that each 1-quasiconformal map defined on a domain of such groups is the restriction of the composition of a standard dilation, a left translation, and an isometric graded isomorphism. In [9], we showed the CR property of 1-quasiconformal mappings on the Engel group and obtained the identity component of the group of 1-quasiconformal mappings. For other deformations of CR mappings between the nilpotent Lie group of step two and their Beltrami equations, one can refer to [10]. In this paper, we will investigate the 1-quasiconformal mappings on Goursat groups, which are stratified nilpotent Lie groups including Engel groups and a large class of high step Carnot groups.

A Goursat group is a nilpotent group endowed with a Goursat distribution $D$. A distribution $D$ of corank $s \geq 2$ on a manifold $M$ is Goursat if the subsheaves $D^{i}$ of the tangent bundle defined inductively by $D^{i+1}=\left[D^{i}, D^{i}\right](i=$ $1,2, \ldots, s ; D^{1}=D ;\left[D^{i}, D^{i}\right]$ denotes the sheaf of vector fields generated by $D^{i}$ and the Lie brackets $[X, Y], X, Y \in D^{i}$ ) correspond to distributions; that is, they have constant rank, and this rank is rank $D^{i+1}=\operatorname{rank} D^{i}+1, i=1,2, \ldots, s[11]$. By a distribution of rank $d$ we understand any subbundle of linear dimension $d$ in the tangent bundle TM; its corank is $\operatorname{dim} M-d$.

Our model of Goursat groups is $G_{n}$ in [12], which takes $\mathbb{R}^{n+1}$ as the underlying space with the group multiplication

$$
\begin{aligned}
& \left(x_{0}, x_{1}, \ldots, x_{n}\right)\left(x_{0}^{\prime}, x_{1}^{\prime}, \ldots, x_{n}^{\prime}\right) \\
& =\left(x_{0}+x_{0}^{\prime}, x_{1}+x_{1}^{\prime}, x_{2}+x_{2}^{\prime}\right. \\
& \quad+x_{0} x_{1}^{\prime}, \ldots, x_{n}+x_{n}^{\prime}+x_{0} x_{n-1}^{\prime} \\
& \left.\quad+\frac{1}{2} x_{0}^{2} x_{n-2}^{\prime}+\cdots+\frac{1}{(n-1) !} x_{0}^{n-1} x_{1}^{\prime}\right) .
\end{aligned}
$$


It is easy to see that its Lie algebra of left-invariant vector fields is given by

$$
\begin{aligned}
& X_{0}=\frac{\partial}{\partial x_{0}}, \\
& X_{j}=\frac{\partial}{\partial x_{j}}+x_{0} \frac{\partial}{\partial x_{j+1}}+\cdots+\frac{x_{0}^{n-j}}{(n-j) !} \frac{\partial}{\partial x_{n}}, \quad j=1, \ldots, n .
\end{aligned}
$$

Then $X_{0}, X_{1}, \ldots, X_{n}$ span the tangent space everywhere and the only nontrivial commutation relations among these vector fields are

$$
\left[X_{0}, X_{j}\right]=X_{j+1}, \quad j=1, \ldots, n-1 .
$$

We use $\mathfrak{g}$ to denote the Lie algebra and it splits into the direct sum

$$
\mathfrak{g}=V_{1} \oplus V_{2} \oplus \cdots \oplus V_{n}
$$

where the vector space $V_{1}$ is spanned by the fields $X_{0}, X_{1}$ and the space $V_{j}, j=2, \ldots, n$, is spanned by $X_{j}$, respectively. It is clear to see that when $n=3, G_{n}$ is Engel group.

Let $d_{c}(\cdot, \cdot)$ be the Carnot-Carathéodory distance on the Goursat group. A homeomorphism $f: \Omega \rightarrow \Omega^{\prime}$ between domains on the Goursat group $G$ is quasiconformal if

$$
\begin{aligned}
H(p) & =\limsup _{r \rightarrow 0} H(p, r) \\
& =\limsup _{r \rightarrow 0} \frac{\max _{d_{c}(p, q)=r} d_{c}(f(p), f(q))}{\min _{d_{c}(p, q)=r} d_{c}(f(p), f(q))}, \quad p \in \Omega,
\end{aligned}
$$

is uniformly bounded. If in addition $\|H\|_{\infty}=\sup _{p \in \Omega} H(p) \leq$ $K$ the homeomorphism $f$ is $K$-quasiconformal.

On $\mathbb{R}^{2}$, a 1-quasiconformal mapping is holomorphic or antiholomorphic. If we identify the Heisenberg group with a quadratic hypersurface in $\mathbb{C}^{n}, 1$-quasiconformal mapping on the quadratic hypersurface is the restriction of a holomorphic or antiholomorphic mapping on $\mathbb{C}^{n}[3]$. This can be viewed as the higher dimensional case of the holomorphicity of 1-quasiconformal mapping. This phenomenon happens for Goursat group.

A CR manifold is a smooth manifold $M$ equipped with a distribution $H \subset T M$ of even rank $2 n$ and a complex structure as endomorphisms $J_{p}: H_{p} \rightarrow H_{p}$ with $J_{p}{ }^{2}=-\mathrm{id}$. Denote

$$
\begin{aligned}
& T_{1,0} M=\operatorname{ker}\left(J_{p}-\sqrt{-1} \mathrm{id}\right), \\
& T_{0,1} M=\operatorname{ker}\left(J_{p}+\sqrt{-1} \mathrm{id}\right) .
\end{aligned}
$$

A CR manifold is supposed to satisfy the following integrability condition: $\left[Z_{1}, Z_{2}\right] \in C^{\infty}\left(T_{1,0} M\right)$, if $Z_{1}, Z_{2} \in C^{\infty}\left(T_{1,0} M\right)$. The number $n$ is called $C R$ dimension of $M$ and $k=\operatorname{dim} M-$ $2 n$ is called the codimension of $M$. A smooth mapping $f$ : $M_{1} \rightarrow M_{2}$ is called $C R$ if

$$
f_{*} T_{1,0} M_{1} \subset T_{1,0} M_{2}
$$

CR manifolds appear naturally as embedded real submanifolds of complex manifolds. The distribution $H$ is then defined as $H=T M \cap i T M$ and $J$ is the restriction of the complex structure in the ambient complex manifold to $H$. We recall that, for a manifold $M=\left\{\rho_{1}=0, \rho_{2}=0, \ldots, \rho_{k}=0\right\}$ with $\bar{\partial} \rho_{1} \wedge \bar{\partial} \rho_{2} \wedge \cdots \wedge \bar{\partial} \rho_{k} \neq 0$, the induced CR structure on $M$ is given by $T_{1,0} M=T_{1,0} \mathbb{C}^{n} \cap \mathbb{C} T M=\left\{Z \in T_{1,0} \mathbb{C}^{n} \mid Z \rho_{1}=\right.$ $\left.0, Z \rho_{2}=0, \ldots, Z \rho_{k}=0\right\}$, and $\operatorname{dim} T_{1,0} M=n-k$.

The Goursat group $G_{n}$ can be realized as the real submanifold of $\mathbb{C}^{n}$ :

$$
\begin{array}{r}
S_{n}=\left\{\left(z, w_{2}, \ldots, w_{n}\right) \in \mathbb{C}^{n} \mid \operatorname{Im} w_{k}=-\frac{1}{2^{k} k !}\left(z^{k}+\bar{z}^{k}\right)\right. \\
-\frac{1}{2^{k}} \sum_{j=1}^{k-1} \frac{1}{j !(k-j) !} z^{j} \bar{z}^{k-j}, \\
k=2, \ldots, n\},
\end{array}
$$

where $z=x+i y, w_{j}=u_{j}+i v_{j}, j=2, \ldots, n$, by the diffeomorphism $T: S_{n} \rightarrow G_{n}$ defined by

$$
T\left(z, w_{2}, \ldots, w_{n}\right)=\left(z, \operatorname{Re} w_{2}, \ldots, \operatorname{Re} w_{n}\right) .
$$

It is easy to check that

$$
Z=\frac{\partial}{\partial z}-i \operatorname{Re} z \frac{\partial}{\partial w_{2}}-\cdots-\frac{i}{(n-1) !}(\operatorname{Re} z)^{n-1} \frac{\partial}{\partial w_{n}},
$$

where $\partial / \partial z=(1 / 2)((\partial / \partial x)-i(\partial / \partial y)), \partial / \partial w_{j}=(1 / 2)$ $\left(\left(\partial / \partial u_{j}\right)-i\left(\partial / \partial v_{j}\right)\right), j=2, \ldots, n$, is the complex tangent vector of $S_{n}$, and we can define the standard CR structure on $S_{n}$ by $T_{1,0} S_{n}=\mathbb{C} Z$ and $\operatorname{dim} T_{1,0} S_{n}=1$. We can also define quasiconformal mappings on $S_{n}$ by (5) with respect to its Carnot-Carathéodory distance defined in Section 2. There is one to one corresponding between 1-quasiconformal mappings on $G_{n}$ and $S_{n}$ via the diffeomorphism $T$. Therefore, if we want to study 1-quasiconformal mappings on $G_{n}$, we can just study the 1-quasiconformal mappings on $S_{n}$. For further details see Section 2.

Denote

$$
\mathscr{P}=\left\{\left(x_{0}, x_{1}, \ldots, x_{n}\right) \in G_{n} \mid x_{2}=x_{3}=\cdots=x_{n}=0\right\} .
$$

We show that 1-quasiconformal mappings between domains on Goursat group $G_{n}$ whose Pansu differentials preserve $\mathscr{P}$ are CR or anti-CR.

Theorem 1. Let $\Omega$ be a domain in $S_{n}$. Suppose that $f=$ $\left(f_{1}, \ldots, f_{n}\right): \Omega \rightarrow f(\Omega)$ is a 1-quasiconformal mapping with $D \widetilde{f}(p)$ preserving $\mathscr{P}$; then $f$ is $C R$ or anti-CR; that is, either

$$
\bar{Z} f_{k}=0, \quad k=1,2, \ldots, n,
$$

or

$$
Z f_{k}=0, \quad k=1,2, \ldots, n,
$$

where $\tilde{f}=T \circ f \circ T^{-1}$ and $p$ is some P-differential point. 
Remark 2. By this theorem the determination of 1-quasiconformal mappings is reduced to the determination of CR automorphisms of a submanifold in $\mathbb{C}^{n}$. According to the extension theorem (Theorem 1.4 in [13]), locally, the smooth CR automorphisms of $S_{n}$ are holomorphic automorphisms of $S_{n}$, which is also an interesting problem.

Remark 3. In the cases of Heisenberg group and Engel group, the condition of $D \widetilde{f}(p)$ preserving $\mathscr{P}$ in Theorem 1 can be omitted. However, by a similar method as in those cases, we cannot remove it, since it is inconvenient to use the expressions of Baker-Campbell-Hausdorff formula for groups of higher step.

\section{Preliminaries}

There is a natural homogeneous norm $\|\cdot\|$ on $G_{n}$, given by

$$
\left\|\left(x_{0}, x_{1}, \ldots, x_{n}\right)\right\|^{2 n !}=\left(x_{0}^{2}+x_{1}^{2}\right)^{n !}+\sum_{k=2}^{n}\left|x_{k}\right|^{2 n ! / k},
$$

and an associated pseudometric $d$, given by

$$
d(p, q)=\left\|q^{-1} p\right\|
$$

for $p, q \in G_{n}$. The metric is left-invariant and is related to the dilations by the formula

$$
d\left(\delta_{c} p, \delta_{c} q\right)=c d(p, q)
$$

where

$$
\delta_{c}\left(x_{0}, x_{1}, \ldots, x_{n}\right)=\left(c x_{0}, c x_{1}, c^{2} x_{2}, \ldots, c^{n} x_{n}\right), \quad c \in \mathbb{R}^{+},
$$

denotes the dilation on $G_{n}$.

The horizontal tangent space at $p \in G_{n}$ is a subspace $H_{p} G_{n}$ of the tangent space $T_{p} G_{n}$, and it is spanned by the vector fields $X_{0}(p)$ and $X_{1}(p)$. An absolutely continuous curve $\gamma$ : $[0,1] \rightarrow G_{n}$ is horizontal if its tangent vectors $\dot{\gamma}(t), t \in[0,1]$, lie in the horizontal tangent space $H_{\gamma(t)} G_{n}$. By Chow [14], any given two points $p, q \in G_{n}$ can be connected by a horizontal curve.

On $H_{p} G_{n}$, we fix a quadric form $\langle\cdot, \cdot\rangle_{G}$ with respect to which the vector fields $X_{0}(p), X_{1}(p)$ are orthonormal at every point $p \in G_{n}$ and satisfy

$$
\begin{aligned}
\left|\alpha X_{0}(p)+\beta X_{1}(p)\right|_{G}^{2} & \\
= & \left\langle\alpha X_{0}(p)+\beta X_{1}(p), \alpha X_{0}(p)+\beta X_{1}(p)\right\rangle_{G} \\
= & \alpha^{2}+\beta^{2},
\end{aligned}
$$

where $\alpha, \beta \in \mathbb{R} .\langle\cdot, \cdot\rangle_{G}$ is called infinitesimal CarnotCarathéodory $(C-C)$ metric. Then the length $l(\gamma)$ of the curve $\gamma$ is defined by

$$
l(\gamma)=\int_{0}^{1}\langle\dot{\gamma}(t), \dot{\gamma}(t)\rangle_{G}^{1 / 2} d t
$$

The Carnot-Carathéodory distance $(C-C$ distance $) d_{c}(p, q)$ is the infimum of the lengths of all absolutely continuous horizontal curves connecting $p$ and $q$ :

$$
d_{c}(p, q)=\inf _{\gamma} l(\gamma)
$$

According to [15], this metric is equivalent to the pseudometric; that is,

$$
C_{1} d(x, y) \leq d_{c}(x, y) \leq C_{2} d(x, y), \quad \forall x, y \in G_{n},
$$

for suitable constants $C_{1}, C_{2}$.

For the manifold $S_{n}$, the horizontal tangent space at $x \in S_{n}$ is $H_{x} S_{n}=\operatorname{span}_{\mathbb{R}}\{\operatorname{Re} Z(x), \operatorname{Im} Z(x)\}$. On $H_{x} S_{n}$, we fix a quadric form $\langle\cdot, \cdot\rangle_{S}$ with respect to which $2 \operatorname{Re} Z(x)$, $2 \operatorname{Im} Z(x)$ are orthonormal at every point $x \in S_{n}$ and satisfy

$$
\begin{aligned}
\mid a \operatorname{Re} & Z(x)+\left.b \operatorname{Im} Z(x)\right|_{S} ^{2} \\
& =\langle a \operatorname{Re} Z(x)+b \operatorname{Im} Z(x), a \operatorname{Re} Z(x)+b \operatorname{Im} Z(x)\rangle_{S} \\
& =\frac{a^{2}+b^{2}}{4},
\end{aligned}
$$

where $a, b \in \mathbb{R}$. The C-C distance on $S_{n}$ can be defined as above.

By definition (8), $S_{n}$ in $\mathbb{C} \times \mathbb{C}^{n-1}$ corresponding to $G_{n}$ is defined by the equation

$$
\rho_{1}=\rho_{2}=\cdots=\rho_{n-1}=0
$$

where

$$
\begin{aligned}
\rho_{1}\left(z, w_{2}, \ldots, w_{n}\right)= & \frac{w_{2}-\bar{w}_{2}}{2 i}+\frac{1}{4} \operatorname{Re} z^{2}+\frac{1}{4}|z|^{2} \\
\rho_{2}\left(z, w_{2}, \ldots, w_{n}\right)= & \frac{w_{3}-\bar{w}_{3}}{2 i}+\frac{1}{8} \operatorname{Re} z^{2} \bar{z}+\frac{1}{24} \operatorname{Re} z^{3} \\
& \vdots \\
\rho_{n-1}\left(z, w_{2}, \ldots, w_{n}\right)= & \frac{w_{n}-\bar{w}_{n}}{2 i}+\frac{1}{2^{n}} \sum_{j=1}^{n-1} \frac{1}{j !(n-j) !} z^{j} \bar{z}^{n-j} \\
& +\frac{1}{2^{n} n !}\left(z^{n}+\bar{z}^{n}\right) .
\end{aligned}
$$

It is direct to check that

$$
\bar{\partial} \rho_{1} \wedge \bar{\partial} \rho_{2} \wedge \cdots \wedge \bar{\partial} \rho_{n-1} \neq 0
$$

And the vector field $Z$ defined by (10) satisfies

$$
Z \rho_{j}=0, \quad j=1, \ldots, n-1 \text {. }
$$

That is, $Z$ is a complex tangent vector field of $S_{n}$, and $S_{n}$ is a CR manifold with the induced CR structure $T_{1,0} S_{n}=\mathbb{C} Z$. We obtain the following relation between $S_{n}$ and $G_{n}$. 
Proposition 4. $S_{n}$ is CR diffeomorphic and isometric to $G_{n}$ via $T$ defined by (9); that is, $T$ and $T^{-1}$ are $C R$ and isometric.

Proof. We can denote the point in $G_{n}$ as $\left(z, x_{2}, x_{3}, \ldots, x_{n}\right)$ where $z=x_{0}+i x_{1}$, and then the group multiplication of $G_{n}$ can be written in the following form:

$$
\begin{aligned}
& \left(z, x_{2}, \ldots, x_{n}\right)\left(z^{\prime}, x_{2}^{\prime}, \ldots, x_{n}^{\prime}\right) \\
& =\left(z+z^{\prime}, x_{2}+x_{2}^{\prime}+(\operatorname{Re} z) \operatorname{Im} z^{\prime}, \ldots, x_{n}\right. \\
& +x_{n}^{\prime}+(\operatorname{Re} z) x_{n-1}^{\prime}+\frac{1}{2}(\operatorname{Re} z)^{2} x_{n-2}^{\prime} \\
& \left.+\cdots+\frac{1}{(n-1) !}(\operatorname{Re} z)^{n-1} \operatorname{Im} z^{\prime}\right) .
\end{aligned}
$$

Then the complex horizontal space is spanned by $Z_{G}, \bar{Z}_{G}$, where

$$
\begin{aligned}
Z_{G} & =\frac{1}{2}\left(X_{0}-i X_{1}\right) \\
& =\frac{\partial}{\partial z}-\frac{i}{2}(\operatorname{Re} z) \frac{\partial}{\partial x_{2}}-\cdots-\frac{i}{2(n-1) !}(\operatorname{Re} z)^{n-1} \frac{\partial}{\partial x_{n}}
\end{aligned}
$$

here $\partial / \partial z=(1 / 2)\left(\left(\partial / \partial x_{0}\right)-i\left(\partial / \partial x_{1}\right)\right)$.

We claim that, under this diffeomorphism $T$ defined by (9), the complex tangent vector fields $Z, \bar{Z}$ of $S_{n}$ are mapped onto $Z_{G}$ and $\bar{Z}_{G}$, respectively. In fact, for any $f \in C^{k}\left(G_{n}\right)$ and $q=\left(z, w_{2}, \ldots, w_{n}\right) \in S_{n}$,

$$
\begin{aligned}
& \left(T_{*} Z\right) f(q) \\
& \quad=Z(f \circ T)(q) \\
& \quad=\left[\frac{\partial}{\partial z}-i \operatorname{Re} z \frac{\partial}{\partial w_{2}}-\cdots-\frac{i}{(n-1) !}(\operatorname{Re} z)^{n-1} \frac{\partial}{\partial w_{n}}\right] \\
& {\left[f\left(z, \bar{z}, \frac{w_{2}+\bar{w}_{2}}{2}, \ldots, \frac{w_{n}+\bar{w}_{n}}{2}\right)\right]} \\
& \quad=\frac{\partial f}{\partial z}-i \frac{\operatorname{Re} z}{2} \frac{\partial f}{\partial x_{2}}-\cdots-\frac{i}{2(n-1) !}(\operatorname{Re} z)^{n-1} \frac{\partial f}{\partial x_{n}} \\
& \quad=Z_{G} f(T(q)) .
\end{aligned}
$$

Therefore,

$$
T_{*} Z=Z_{G}
$$

Similarly

$$
T_{*} \bar{Z}=\bar{Z}_{G}
$$

It is clear to see that $T^{-1}: G_{n} \rightarrow S_{n}$ is

$$
T^{-1}\left(z, x_{2}, \ldots, x_{n}\right)=\left(z, x_{2}+i v_{2}, \ldots, x_{n}+i v_{n}\right) \text {, }
$$

where

$$
\begin{array}{r}
v_{k}=-\frac{1}{2^{k} k !}\left(z^{k}+\bar{z}^{k}\right)-\frac{1}{2^{k}} \sum_{j=1}^{k-1} \frac{1}{j !(k-j) !} z^{j} \bar{z}^{k-j}, \\
k=2, \ldots, n .
\end{array}
$$

Similarly, we find

$$
T_{*}^{-1} Z_{G}=Z, \quad T_{*}^{-1} \bar{Z}_{G}=\bar{Z} .
$$

Hence, $T, T^{-1}$ are CR and by (30), (31), and (34) together with (18) and (22), we get $\left|T_{*} X\right|_{G}=|X|_{S}$ for any $X \in H_{q} S_{n}$ and $\left|T_{*}^{-1} Y\right|_{S}=|Y|_{G}$ for any $Y \in H_{p} G_{n}$, so $T, T^{-1}$ preserve C-C distance. This proves the proposition.

\section{P-Differential of 1-Quasiconformal Mappings on $G_{n}$}

The notion of differential of a map between domains in Carnot group is due to Pansu [16]. Let $\tau_{p}(h)=p h$ denote left-translation on $G_{n}$. The mapping $f: \Omega \rightarrow \Omega^{\prime}\left(\Omega, \Omega^{\prime}\right.$ are domains in $G_{n}$ ) is called $p$-differentiable at $p \in \Omega$ if, for $c \rightarrow 0$, the mappings

$$
\delta_{c}^{-1} \circ \tau_{f(p)}^{-1} \circ f \circ \tau_{p} \circ \delta_{c}
$$

converge locally uniformly to a homomorphism $D f(p)$ of the group $G_{n}$, which is called Pansu differential (P-differential).

The Pansu differential $D f(p)$ is a Carnot homomorphism. Denote by $D f_{*}(p)$ the induced Lie algebra homomorphism of the group homomorphism $D f(p)$. According to [17], $D f_{*}(p)$ is grading-preserving. As a result of [16] or [18], quasiconformal mappings between domains in Carnot group are $P$-differentiable a.e. The result is valid on the group $G_{n}$ as it is a Carnot group.

Define the bilinear form $B$ on $\mathfrak{g}$ by

$$
\left[X_{j}, X_{k}\right]=B\left(X_{j}, X_{k}\right) X_{2}, \quad j, k=0,1
$$

Then by $\left[X_{0}, X_{1}\right]=X_{2}$, the corresponding matrix is

$$
B=\left(\begin{array}{cc}
0 & 1 \\
-1 & 0
\end{array}\right) \text {. }
$$

Let $f$ be a 1-quasiconformal mapping between domains in $G_{n}$; since $D f_{*}(p)$ is grading-preserving, then $D f_{*}(p)$ maps $V_{j}(j=1, \ldots, n)$ onto itself, respectively. In particular $V_{1}=$ $\operatorname{span}\left\{X_{0}, X_{1}\right\}$, so for any $X \in V_{1}$,

$$
D f_{*}(p) X=S X,
$$

for some $2 \times 2$ matrix $S$. Since $V_{2}=\mathbb{R} X_{2}$, then

$$
D f_{*}(p) X_{2}=\lambda X_{2}
$$

for some $\lambda$. 
Proposition 5. The matrix $S$ above can be written as the following form:

$$
S=\left(\begin{array}{cc}
c \cos \theta & c \sin \theta \\
-\frac{\lambda}{c} \sin \theta & \frac{\lambda}{c} \cos \theta
\end{array}\right),
$$

for some $\theta \in[0,2 \pi)$, where $c=\left|D f_{*}(p) X\right|_{G}$ for any $X \in V_{1}$ with $|X|_{G}=1, \lambda^{2} / c^{4}=1 ; \lambda, \theta, c$ depend on $p \in G_{n}$.

Proof. Since $D f_{*}(p) X=S X, X \in V_{1}$, now suppose

$$
S=\left(\begin{array}{ll}
s_{11} & s_{12} \\
s_{21} & s_{22}
\end{array}\right)
$$

then

$$
D f_{*}(p) X_{k}=s_{1 k} X_{0}+s_{2 k} X_{1}, \quad k=0,1 .
$$

Because $D f_{*}(p)$ is Lie algebra homomorphism, that is,

$$
\left[D f_{*}(p) X_{k}, D f_{*}(p) X_{l}\right]=D f_{*}(p)\left[X_{k}, X_{l}\right],
$$

together with $D f_{*}(p) X_{2}=\lambda X_{2}$, we find that

$$
B\left(s_{1 k} X_{0}+s_{2 k} X_{1}, s_{1 l} X_{0}+s_{2 l} X_{1}\right)=\lambda B\left(X_{k}, X_{l}\right),
$$

namely,

$$
S^{T} B S=\lambda B .
$$

By Lemma 3.3 in [19], for the 1-quasiconformal mapping $f$, we have

$$
\frac{\max _{X \in V_{1},|X|_{G}=1}\left|D f_{*}(p) X\right|_{G}}{\min _{X \in V_{1},|X|_{G}=1}\left|D f_{*}(p) X\right|_{G}}=1,
$$

where $|\cdot|_{G}$ is defined by (18), that is,

$$
\frac{\max _{X \in V_{1},|X|_{G}=1}|S X|_{G}}{\min _{X \in V_{1},|X|_{G}=1}|S X|_{G}}=1 .
$$

Therefore, there exists $c>0$, such that

$$
|S X|_{G}=c \text {, }
$$

for any $X \in V_{1},|X|_{G}=1$. Then $(1 / c) S$ is an orthogonal matrix. Together with (45), we have

$$
B S=\frac{\lambda}{c^{2}} S B,
$$

that is,

$$
\left(\begin{array}{cc}
0 & 1 \\
-1 & 0
\end{array}\right)\left(\begin{array}{ll}
s_{11} & s_{12} \\
s_{21} & s_{22}
\end{array}\right)=\frac{\lambda}{c^{2}}\left(\begin{array}{ll}
s_{11} & s_{12} \\
s_{21} & s_{22}
\end{array}\right)\left(\begin{array}{cc}
0 & 1 \\
-1 & 0
\end{array}\right) .
$$

Thus

$$
\begin{aligned}
& s_{21}=-\frac{\lambda}{c^{2}} s_{12}, \\
& s_{22}=\frac{\lambda}{c^{2}} s_{11}, \\
& s_{11}=\frac{\lambda}{c^{2}} s_{22}, \\
& s_{12}=-\frac{\lambda}{c^{2}} s_{21} .
\end{aligned}
$$

Therefore, $\lambda^{2} / c^{4}=1$. Note that $((1 / c) S)^{T}((1 / c) S)=I$, where $I$ is the $2 \times 2$ unit matrix; hence

$$
\begin{aligned}
s_{11}^{2}+s_{12}^{2} & =c^{2}, \\
s_{21}^{2}+s_{22}^{2} & =c^{2}, \\
s_{11} s_{21}+s_{12} s_{22} & =0 .
\end{aligned}
$$

From (51), (52) and setting $s_{11}=c \cos \theta, \theta \in[0,2 \pi)$, we have (40). The result follows.

We get the following relation between $P$-differential and the usual derivatives. For the corresponding result on Heisenberg group see p.31 of [3]. In the following, the letter $C$ will be used to denote various constants, and the various uses of the letter do not, however, denote the same constant.

Proposition 6. Suppose $f$ is a quasiconformal mapping between domains in $G_{n}$ with $f(0)=0$, and if $f$ is $P$ differentiable at 0 and $D f(0)$ preserves the plan $\mathscr{P}$ in (11), then $f$ is differentiable in the usual sense at 0 along the horizontal directions and

$$
D f_{*}(0) \partial_{x_{j}}=f_{* 0} \partial_{x_{j}}, \quad j=0,1,
$$

where $f_{* 0} \partial_{x_{j}}=\sum_{k=0}^{n}\left(\partial f_{k} / \partial x_{j}\right)(0) \partial_{x_{k}}$ and $D f_{*}(0)$ is the $P$ derivative of $f$ at 0 .

Proof. Since $f$ is $P$-differentiable at 0 , by definition, there exists $0<\delta<1$ such that, for $c<\delta$ and $\left\|\left(z, x_{2}, \ldots, x_{n}\right)\right\| \leq 1$,

$$
\begin{aligned}
\|\left(D f(0)\left(z, x_{2}, \ldots, x_{n}\right)\right)^{-1} \\
\quad \times\left(\delta_{c}^{-1} \circ \tau_{f(0)}^{-1} \circ f \circ \tau_{0} \circ \delta_{c}\left(z, x_{2}, \ldots, x_{n}\right)\right) \| \\
=\|\left(D f(0)\left(z, x_{2}, \ldots, x_{n}\right)\right)^{-1} \\
\quad \times\left(\delta_{c}^{-1} \circ f \circ \delta_{c}\left(z, x_{2}, \ldots, x_{n}\right)\right) \|<\varepsilon .
\end{aligned}
$$

Recall that the homomorphism $D f(0)$ commutes with $\delta_{c}$; then for points $(z /|z|, 0, \ldots, 0)$ in particular, we have

$$
\begin{aligned}
& \left\|\delta_{c}^{-1}\left(\left(D f(0)\left(c \frac{z}{|z|}, 0, \ldots, 0\right)\right)^{-1} f\left(c \frac{z}{|z|}, 0, \ldots, 0\right)\right)\right\| \\
& =\frac{1}{c}\left\|\left(D f(0)\left(c \frac{z}{|z|}, 0, \ldots, 0\right)\right)^{-1} f\left(c \frac{z}{|z|}, 0, \ldots, 0\right)\right\|<\varepsilon .
\end{aligned}
$$

Therefore

$$
\begin{array}{r}
\frac{1}{|z|}\left\|(D f(0)(z, 0, \ldots, 0))^{-1} f(z, 0, \ldots, 0)\right\|<\varepsilon, \\
\text { if }|z|<\delta .
\end{array}
$$

Now compare this with Euclidean distances. Since $D f(0)$ preserves the plane $\mathscr{P}$, that is,

$$
\operatorname{Df}(0)(z, 0, \ldots, 0)=(\widetilde{z}, 0, \ldots, 0)=\left(\tilde{x}_{0}, \tilde{x}_{1}, 0, \ldots, 0\right)
$$


for some $\widetilde{z}=\left(\tilde{x}_{0}, \tilde{x}_{1}\right) \in \mathbb{R}^{2}$, then

$$
\begin{aligned}
(D f(0) & (z, 0, \ldots, 0))^{-1} \\
\quad & =\left(\tilde{x}_{0}, \tilde{x}_{1}, 0, \ldots, 0\right)^{-1} \\
& =\left(-\tilde{x}_{0},-\tilde{x}_{1}, \tilde{x}_{0} \tilde{x}_{1},-\frac{\tilde{x}_{0}^{2}}{2} \tilde{x}_{1}, \ldots, \frac{(-1)^{n} \tilde{x}_{0}^{n-1}}{(n-1) !} \tilde{x}_{1}\right) .
\end{aligned}
$$

Assume that $\left(x_{0}^{\prime}, x_{1}^{\prime}, x_{2}^{\prime}, \ldots, x_{n}^{\prime}\right)=f(z, 0, \ldots, 0)$; we get

$$
\begin{aligned}
& \left\|(D f(0)(z, 0, \ldots, 0))^{-1} f(z, 0, \ldots, 0)\right\|^{2 n !} \\
& =\|\left(-\tilde{x}_{0},-\tilde{x}_{1}, \tilde{x}_{0} \tilde{x}_{1},-\frac{\tilde{x}_{0}^{2}}{2} \tilde{x}_{1}, \ldots, \frac{(-1)^{n} \tilde{x}_{0}^{n-1}}{(n-1) !} \tilde{x}_{1}\right) \\
& \quad \times\left.\left(x_{0}^{\prime}, x_{1}^{\prime}, x_{2}^{\prime}, \ldots, x_{n}^{\prime}\right)\right|^{2 n !} \\
& =\left(\left|x_{0}^{\prime}-\tilde{x}_{0}\right|^{2}+\left|x_{1}^{\prime}-\tilde{x}_{1}\right|^{2}\right)^{n !}+\left|x_{2}^{\prime}-\tilde{x}_{0}\left(x_{1}^{\prime}-\tilde{x}_{1}\right)\right|^{2 n ! / 2} \\
& +\left|x_{3}^{\prime}-\tilde{x}_{0} x_{2}^{\prime}+\frac{\left(-\tilde{x}_{0}\right)^{2}}{2}\left(x_{1}^{\prime}-\tilde{x}_{1}\right)\right|^{2 n ! / 3}+\cdots \\
& +\mid x_{n}^{\prime}-\tilde{x}_{0} x_{n-1}^{\prime}+\frac{\left(-\tilde{x}_{0}\right)^{2}}{2} x_{n-2}^{\prime}+\cdots \\
& \quad+\left.\frac{\left(-\tilde{x}_{0}\right)^{n-1}}{(n-1) !}\left(x_{1}^{\prime}-\tilde{x}_{1}\right)\right|^{2 n ! / n} \\
& <\varepsilon^{2 n !}|z|^{2 n !} .
\end{aligned}
$$

It follows that

$$
\begin{aligned}
& \left|x_{0}^{\prime}-\tilde{x}_{0}\right|^{2}+\left|x_{1}^{\prime}-\tilde{x}_{1}\right|^{2}<\varepsilon^{2}|z|^{2}, \\
& \left|x_{2}^{\prime}-\tilde{x}_{0}\left(x_{1}^{\prime}-\tilde{x}_{1}\right)\right|<\varepsilon^{2}|z|^{2}, \\
& \left|x_{3}^{\prime}-\tilde{x}_{0} x_{2}^{\prime}+\frac{\left(-\tilde{x}_{0}\right)^{2}}{2}\left(x_{1}^{\prime}-\tilde{x}_{1}\right)\right|<\varepsilon^{3}|z|^{3}, \\
& \vdots \\
& \mid x_{n}^{\prime}-\tilde{x}_{0} x_{n-1}^{\prime}+\frac{\left(-\tilde{x}_{0}\right)^{2}}{2} x_{n-2}^{\prime}+\cdots \\
& +\left.\frac{\left(-\tilde{x}_{0}\right)^{n-1}}{(n-1) !}\left(x_{1}^{\prime}-\tilde{x}_{1}\right)\left|<\varepsilon^{n}\right| z\right|^{n} .
\end{aligned}
$$

Therefore,

$$
\left|x_{0}^{\prime}-\tilde{x}_{0}\right|<\varepsilon|z|, \quad\left|x_{1}^{\prime}-\tilde{x}_{1}\right|<\varepsilon|z|,
$$

$$
\left|x_{2}^{\prime}\right|<\varepsilon^{2}|z|^{2}+\varepsilon\left|\tilde{x}_{0}\right||z| \text {, }
$$

$$
\left|x_{3}^{\prime}\right|<\varepsilon^{3}|z|^{3}+\left|\tilde{x}_{0}\right|\left|x_{2}^{\prime}\right|+\frac{\left(-\tilde{x}_{0}\right)^{2}}{2} \varepsilon|z|
$$

$$
\begin{aligned}
\left|x_{n}^{\prime}\right|< & \varepsilon^{n}|z|^{n}+\left|\tilde{x}_{0}\right|\left|x_{n-1}^{\prime}\right|+\frac{\left(-\tilde{x}_{0}\right)^{2}}{2}\left|x_{n-2}^{\prime}\right|+\cdots \\
& +\varepsilon\left|\frac{\left(-\tilde{x}_{0}\right)^{n-1}}{(n-1) !}\right||z| .
\end{aligned}
$$

From Lemma 3.3 in [19], $D f(0)$ is Lipschitz; then by the equivalence of $d_{c}$ and $d$, we have

$$
\|D f(0)(z, 0, \ldots, 0)\| \leq \operatorname{CLip}_{f}(0)\|(z, 0, \ldots, 0)\|,
$$

for some $C>0$, namely,

$$
\left|\tilde{x}_{0}\right| \leq\|D f(0)(z, 0, \ldots, 0)\| \leq \operatorname{CLip}_{f}(0)|z|=M_{1}|z| .
$$

Since $f$ is continuous, then when $|z|<\delta<1$, we have

$$
\left|\left(x_{0}^{\prime}, x_{1}^{\prime}, x_{2}^{\prime}, \ldots, x_{n}^{\prime}\right)\right| \leq|f(z, 0, \ldots, 0)|<M_{2}
$$

where $M_{2}$ is a positive constant. Now substituting (64) and (65) into (62) and then by iteration, we obtain

$$
\begin{aligned}
&\left|x_{2}^{\prime}\right|<\varepsilon^{2}|z|^{2}+\varepsilon M_{1}|z|^{2}<C \varepsilon|z|^{2}, \\
&\left|x_{3}^{\prime}\right|<\varepsilon^{3}|z|^{3}+\left|\tilde{x}_{0}\right|\left|x_{2}^{\prime}\right|+\frac{\left(-\tilde{x}_{0}\right)^{2}}{2} \varepsilon|z| \\
&<\varepsilon^{3}|z|^{3}+C \varepsilon\left|\tilde{x}_{0}\right||z|^{2}+\frac{\left(-\tilde{x}_{0}\right)^{2}}{2} \varepsilon|z|<C \varepsilon|z|^{3}, \\
& \vdots \\
&\left|x_{n}^{\prime}\right|<\varepsilon^{n}|z|^{n}+\left|\tilde{x}_{0}\right|\left|x_{n-1}^{\prime}\right|+\frac{\left(-\tilde{x}_{0}\right)^{2}}{2}\left|x_{n-2}^{\prime}\right|+\cdots \\
& \quad+\left.\varepsilon\left|\frac{\left(-\tilde{x}_{0}\right)^{n-1} \mid}{(n-1) !}\right| z\right|^{n} \\
& \quad<\varepsilon^{2}|z|^{n}+C \varepsilon\left|\tilde{x}_{0}\right||z|^{n-1}+C \varepsilon \frac{\left(-\tilde{x}_{0}\right)^{2}}{2}|z|^{n-2}+\cdots \\
& \quad+C \varepsilon\left|\frac{\left(-\tilde{x}_{0}\right)^{n-1}}{(n-1) !}\right||z| \\
& \quad C \varepsilon|z|^{n} \cdot
\end{aligned}
$$


Then by (61), for any $|z|<\delta<1$, we have

$$
\begin{aligned}
& \frac{1}{|z|}|D f(0)(z, 0, \ldots, 0)-f(z, 0, \ldots, 0)| \\
& \quad=\frac{1}{|z|}\left|\left(\tilde{x}_{0}, \tilde{x}_{1}, 0, \ldots, 0\right)-\left(x_{0}^{\prime}, x_{1}^{\prime}, x_{2}^{\prime}, \ldots, x_{n}^{\prime}\right)\right| \\
& \quad=\frac{1}{|z|}\left(\left|x_{0}^{\prime}-\tilde{x}_{0}\right|^{2}+\left|x_{1}^{\prime}-\tilde{x}_{1}\right|^{2}+\sum_{k=2}^{n}\left|x_{k}^{\prime}\right|^{2}\right)^{1 / 2} \\
& \quad<C \frac{1}{|z|}\left(\varepsilon^{2}|z|^{2}+\sum_{k=2}^{n} \varepsilon^{2}|z|^{2 k}\right)^{1 / 2} \\
& \quad<C \varepsilon .
\end{aligned}
$$

If we let $z=\left(x_{0}, 0\right)$, then

$$
\begin{aligned}
& \frac{1}{\left|x_{0}\right|}\left|D f(0)\left(x_{0}, 0, \ldots, 0\right)-f\left(x_{0}, 0, \ldots, 0\right)\right| \\
& \quad=\left|D f(0)(1,0, \ldots, 0)-\frac{f\left(x_{0}, 0, \ldots, 0\right)}{x_{0}}\right| \\
& \quad<C \varepsilon,
\end{aligned}
$$

namely,

$$
D f(0)(1,0, \ldots, 0)=\lim _{x_{0} \rightarrow 0} \frac{f\left(x_{0}, 0, \ldots, 0\right)-f(0)}{x_{0}}=\frac{\partial f}{\partial x_{0}}(0) \text {. }
$$

Therefore,

$$
D f_{*}(0) \partial_{x_{0}}=\sum_{j=0}^{n} \frac{\partial f_{j}}{\partial x_{0}}(0) \partial_{x_{j}}=f_{* 0} \partial_{x_{0}}
$$

Similarly

$$
D f_{*}(0) \partial_{x_{1}}=\sum_{j=0}^{n} \frac{\partial f_{j}}{\partial x_{1}}(0) \partial_{x_{j}}=f_{* 0} \partial_{x_{1}}
$$

The proposition is proved.

We need the following relation between $P$-differential and the usual tangential mapping. See Proposition 3.2 in [10] for the corresponding result on the Heisenberg group.

Proposition 7. Let $f: \Omega_{1} \rightarrow \Omega_{2}$ be a quasiconformal mapping between domains in the Goursat group $G_{n}$. Suppose $f$ is $P$-differentiable at $p$ and $D f(p)$ preserves the plan $\mathscr{P}$ in (11); then

$$
f_{*}(p) X(p)=\left(\tau_{f(p)}\right)_{* 0}\left[D f_{*}(p) X(0)\right]
$$

for $X \in V_{1}$, where $f_{*}(p) X(p)=\sum_{k=0}^{n} X f_{k}(p) \partial_{x_{k}}$ and $\tau_{q_{*}}$ are the tangential mappings of $\tau_{q}$ for $q \in G_{n}$.
Proof. Suppose $f$ is $P$-differentiable at $p \in G_{n}$; let $F=$ $\tau_{f(p)}^{-1} \circ f \circ \tau_{p}$; then $F$ is also a quasiconformal mapping and $F(0)=0$. Moreover, by Chain rule (Lemma 3.7 in [19]), $F$ is $P$-differentiable at the origin 0 , and

$$
D F(0)=D \tau_{f(p)}^{-1}(f(p)) D f(p) D \tau_{p}(0) .
$$

Since $D \tau_{p}$ is the identity automorphism, then

$$
D F(0)=D f(p) \text {. }
$$

Therefore, $D F(0)$ preserves the plan $\mathscr{P}$.

By Proposition 6, we have

$$
D F_{*}(0) \partial_{x_{j}}=F_{* 0} \partial_{x_{j}}, \quad j=0,1 .
$$

From (2) we can see that $X_{0}(0)=\partial_{x_{0}}$ and $X_{1}(0)=\partial_{x_{1}}$. Therefore,

$$
D F_{*}(0) X_{j}(0)=F_{* 0} X_{j}(0), \quad j=0,1 .
$$

Consequently,

$$
\begin{aligned}
f_{*} & (p) X_{j}(p) \\
& =\left(\tau_{f(p)} \circ F \circ \tau_{p}^{-1}\right)_{* p} X_{j}(p) \\
& =\left(\tau_{f(p)}\right)_{* 0} F_{* 0} X_{j}(0) \\
& =\left(\tau_{f(p)}\right)_{* 0}\left[D F_{*}(0) X_{j}(0)\right] \\
& =\left(\tau_{f(p)}\right)_{* 0}\left[D f_{*}(p) X_{j}(0)\right], \\
& j=0,1 .
\end{aligned}
$$

This completes the proof.

We need the following proposition due to Capogna ([19], Theorem 1.1).

Proposition 8. Suppose that $\Omega_{1}$ and $\Omega_{2}$ are open subsets of Carnot groups $G_{1}$ and $G_{2}$, and $f: \Omega_{1} \rightarrow \Omega_{2}$ is 1quasiconformal. Then $f$ is smooth.

Proof of Theorem 1. Let $f$ be a 1-quasiconformal mapping satisfying the hypothesis in Theorem 1. By Proposition 4, $T, T^{-1}$ defined by (9) and (32) are CR and isometric; then $\tilde{f}=$ $T \circ f \circ T^{-1}$ is a 1-quasiconformal mapping between domains in $G_{n}$. Suppose $p \in G_{n}$ is a $P$-differentiable point of $\widetilde{f}$; then $D \tilde{f}_{*}(p)$ is grading-preserving; that is, $D \tilde{f}_{*}(p) X_{2}=\lambda X_{2}$ for some $\lambda$, and $D \tilde{f}_{*} X=S X, X \in V_{1}$. From Proposition 5, at $p \in G_{n}, S$ has form (40), and $\lambda= \pm c^{2}$, where $c=\left|D \tilde{f}_{*}(p) X\right|_{G}$ for any $X \in V_{1}$ with $|X|_{G}=1$.

In the complexified subspace $\mathbb{C} V_{1}$, the corresponding complex basis is $Z_{G}, \bar{Z}_{G}$, where $Z_{G}$ is as (28). On the matrix level

$$
S \longrightarrow S_{c}=\Theta S \Theta^{-1}
$$


with $\Theta=\left(\begin{array}{cc}1 & i \\ 1 & -i\end{array}\right)$. Then

$$
S_{c}=\left(\begin{array}{ll}
\frac{c^{2}+\lambda}{2 c} e^{-i \theta} & \frac{c^{2}-\lambda}{2 c} e^{i \theta} \\
\frac{c^{2}-\lambda}{2 c} e^{-i \theta} & \frac{c^{2}+\lambda}{2 c} e^{i \theta}
\end{array}\right), \quad \theta \in[0,2 \pi) .
$$

We claim that $D \tilde{f}_{*}(p) Z_{G}=S_{c} Z_{G}, D \tilde{f}_{*}(p) \bar{Z}_{G}=S_{c} \bar{Z}_{G}$. In fact, by (28) and (40), we have

$$
\begin{aligned}
D \tilde{f}_{*} & (p) Z_{G} \\
& =D \tilde{f}_{*}(p)\left[\frac{1}{2}\left(X_{0}-i X_{1}\right)\right] \\
& =\frac{1}{2}\left[c X_{0} \cos \theta-\frac{\lambda}{c} X_{1} \sin \theta-i\left(c X_{0} \sin \theta+\frac{\lambda}{c} X_{1} \cos \theta\right)\right] \\
& =\frac{1}{2}\left[c e^{-i \theta} X_{0}-i \frac{\lambda}{c} e^{-i \theta} X_{1}\right] \\
& =\frac{1}{2} e^{-i \theta}\left[c\left(Z_{G}+\bar{Z}_{G}\right)+\frac{\lambda}{c}\left(Z_{G}-\bar{Z}_{G}\right)\right] \\
& =\frac{1}{2} e^{-i \theta}\left[\left(c+\frac{\lambda}{c}\right) Z_{G}+\left(c-\frac{\lambda}{c}\right) \bar{Z}_{G}\right]=S_{c} Z_{G} .
\end{aligned}
$$

Similarly, $D \tilde{f}_{*}(p) \bar{Z}_{G}=S_{c} \bar{Z}_{G}$.

From Proposition $8, \widetilde{f}$ is smooth, and therefore $f=T^{-1}$ 。 $\tilde{f} \circ T$ is also smooth. We write the 1-quasiconformal mapping $f$ in the form

$$
\begin{aligned}
f\left(z, w_{2}, \ldots, w_{n}\right) & =\left(f_{1}, f_{2}, \ldots, f_{n}\right)\left(z, w_{2}, \ldots, w_{n}\right) \\
& =\left(\omega_{1}, \omega_{2}, \ldots, \omega_{n}\right)
\end{aligned}
$$

with

$$
\begin{aligned}
\operatorname{Im} f_{k}= & -\frac{1}{2^{k} k !}\left(f_{1}^{k}+\bar{f}_{1}^{k}\right) \\
& -\frac{1}{2^{k}} \sum_{j=1}^{k-1} \frac{1}{j !(k-j) !} f_{1}^{j} \bar{f}_{1}^{k-j},
\end{aligned}
$$

where $k=2,3, \ldots, n$. Then

$$
f_{*} Z=U+\bar{V}
$$

where

$$
U=\sum_{k=1}^{n}\left(Z f_{k}\right) \frac{\partial}{\partial \omega_{k}}, \quad \bar{V}=\sum_{k=1}^{n}\left(Z \bar{f}_{k}\right) \frac{\partial}{\partial \bar{\omega}_{k}} .
$$

In fact, for any $\varphi \in C^{k}\left(S_{n}\right)$,

$$
\begin{aligned}
\left(f_{*} Z\right) \varphi & =Z(\varphi \circ f) \\
& =Z\left[\varphi\left(f_{1}, f_{2}, \ldots, f_{n}, \bar{f}_{1}, \bar{f}_{2}, \ldots, \bar{f}_{n}\right)\right] \\
& =\sum_{k=1}^{n} \frac{\partial \varphi}{\partial \omega_{k}} Z f_{k}+\sum_{k=1}^{n} \frac{\partial \varphi}{\partial \bar{\omega}_{k}} Z \bar{f}_{k} \\
& =\left[\sum_{k=1}^{n}\left(Z f_{k}\right) \frac{\partial}{\partial \omega_{k}}+\sum_{k=1}^{n}\left(Z \bar{f}_{k}\right) \frac{\partial}{\partial \bar{\omega}_{k}}\right] \varphi .
\end{aligned}
$$

Let $q=T^{-1}(p) \in S_{n}$; by (30), (34), (72), and (80) together with the left-invariant property of $Z_{G}$,

$$
\begin{aligned}
f_{* q}(Z(q))= & \left(T^{-1} \circ \tilde{f} \circ T\right)_{* q}(Z(q)) \\
= & T_{*}^{-1} \circ \tilde{f}_{*} \circ T_{* q}(Z(q)) \\
= & T_{*}^{-1} \circ \tilde{f}_{* p}\left(Z_{G}(p)\right) \\
= & T_{*}^{-1} \circ \tau_{\tilde{f}(p) * 0} \circ\left[D \tilde{f}_{*}(p) Z_{G}(0)\right] \\
= & T_{* \tilde{f}(p)}^{-1} \circ \tau_{\tilde{f}(p) * 0} \\
& \times\left(\frac{c^{2}+\lambda}{2 c} e^{-i \theta} Z_{G}(0)+\frac{c^{2}-\lambda}{2 c} e^{i \theta} \bar{Z}_{G}(0)\right) \\
= & T_{* \tilde{f}(p)}^{-1}\left(\frac{c^{2}+\lambda}{2 c} e^{-i \theta} Z_{G}(\tilde{f}(p))\right. \\
& \left.+\frac{c^{2}-\lambda}{2 c} e^{i \theta} \bar{Z}_{G}(\tilde{f}(p))\right) \\
= & \frac{c^{2}+\lambda}{2 c} e^{-i \theta} Z(f(q))+\frac{c^{2}-\lambda}{2 c} e^{i \theta} \bar{Z}(f(q)) .
\end{aligned}
$$

Since $f$ is $P$-differentiable a.e., we have

$$
f_{*} Z=\frac{c^{2}+\lambda}{2 c} e^{-i \theta} Z+\frac{c^{2}-\lambda}{2 c} e^{i \theta} \bar{Z}, \quad \text { a.e. }
$$

Consequently, $U, \bar{V}$ in (83) have the following forms

$$
\begin{aligned}
& U=\sum_{k=1}^{n}\left(Z f_{k}\right) \frac{\partial}{\partial \omega_{k}}=\frac{c^{2}+\lambda}{2 c} e^{-i \theta} Z, \quad \text { a.e., } \\
& \bar{V}=\sum_{k=1}^{n}\left(Z \bar{f}_{k}\right) \frac{\partial}{\partial \bar{\omega}_{k}}=\frac{c^{2}-\lambda}{2 c} e^{i \theta} \bar{Z}, \quad \text { a.e. }
\end{aligned}
$$

Hence, if $\lambda=c^{2}$, then $\bar{Z} f_{k}=0, k=1,2, \ldots, n$; if $\lambda=-c^{2}$, then $Z f_{k}=0, k=1,2, \ldots, n$. Since $Z$ and $f$ are smooth, Theorem 1 follows.

Remark 9. The CR automorphism of $G_{n}(n>3)$ is unknown now. It is also very interesting to determine it, since if this can be solved, we can get the 1-quasiconformal mappings on these groups.

\section{Conflict of Interests}

The authors declare that there is no conflict of interests regarding the publication of this paper.

\section{Acknowledgments}

This work was partially supported by NSF of China (Grant nos. 11271175, 11301248 and 11326079), the DYSP, and AMEP of Linyi University. 


\section{References}

[1] G. D. Mostow, Strong Rigidity of Locally Symmetric Spaces, Princeton University Press, Princeton, NJ, USA, 1973.

[2] A. Korányi and H. M. Reimann, "Quasiconformal mappings on the Heisenberg group," Inventiones Mathematicae, vol. 80, no. 2, pp. 309-338, 1985.

[3] A. Korányi and H. M. Reimann, "Foundations for the theory of quasiconformal mappings on the Heisenberg Group," Advances in Mathematics, vol. 111, no. 1, pp. 1-87, 1995.

[4] L. Capogna, "Regularity of Quasi-Linear equations in the Heisenberg group," Communications on Pure and Applied Mathematics, vol. 50, no. 9, pp. 867-889, 1997.

[5] M. Cowling, F. De Mari, A. Korányi, and H. M. Reimann, “Contact and conformal maps in: parabolic geometry I," Geometriae Dedicata, vol. 111, no. 1, pp. 65-86, 2005.

[6] A. Ottazzi and B. Warhurst, "Contact and 1-quasiconformal maps on carnot groups," Journal of Lie Theory, vol. 21, no. 4, pp. 787-811, 2011.

[7] A. Ottazzi and B. Warhurst, "A liouville type theorem for Carnot groups: a case study," in Trends in Harmonic Analysis, pp. 371380, Springer, Milan, Italy, 2013.

[8] X. Xie, "1-quasiconformal maps on Carnot groups with reducible first layer," http://arxiv-web3.library.cornell.edu/abs/ 1312.7487.

[9] Q. Wu and W. Wang, "Conformal mappings and CR mappings on the Engel group," Science in China A: Mathematics, vol. 52, no. 12, pp. 2759-2773, 2009.

[10] W. Wang, "The teichmüller distance on the space of spherical CR structures," Science in China Series A, vol. 49, pp. 1523-1538, 2006.

[11] R. Montgomery and M. Zhitomirskii, "Geometric approach to Goursat flags," Annales de l'Institut Henri Poincaré C: Analyse Non Linéaire, vol. 18, pp. 459-493, 2001.

[12] D. Müller, "Another example in the solvability theory of PDOs with double characteristics," Communications in Partial Differential Equations, vol. 20, no. 11-12, pp. 2165-2186, 1995.

[13] F. Meylan, N. Mir, and D. Zaitsev, "Holomorphic extension of smooth CR-mappings betweenreal-analytic and real-algebraic CR-manifolds," Asian Journal of Mathematics, vol. 7, no. 4, pp. 493-509, 2003.

[14] W.-L. Chow, "Über systeme von liearren partiellen differentialgleichungen erster ordnung," Mathematische Annalen, vol. 117, no. 1, pp. 98-105, 1940.

[15] A. Nagel, E. M. Stein, and S. Wainger, "Balls and metrics defined by vector fields I: basic properties," Acta Mathematica, vol. 155, no. 1, pp. 103-147, 1985.

[16] P. Pansu, Quasiisométries des variétés de courbure négative [Ph.D. thesis], Universityof Paris VII, Paris, France, 1987.

[17] Z. M. Balogh, I. Holopainen, and J. T. Tyson, "Singular solutions, homogeneous norms, and quasiconformal mappings in Carnot groups," Mathematische Annalen, vol. 324, no. 1, pp. 159186, 2002.

[18] S. K. Vodop'yanov, "Differentiability of maps of Carnot groups of Sobolev classes," Matematicheskii Sbornik, vol. 194, pp. 67-86, 2003.

[19] L. Capogna and M. Cowling, "Conformality and $Q$ in carnot groups," Duke Mathematical Journal, vol. 135, no. 3, pp. 455-479, 2006. 


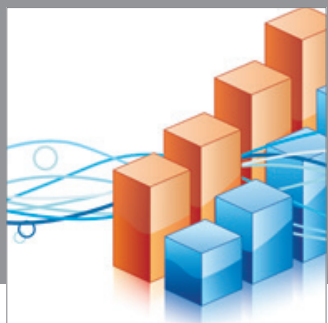

Advances in

Operations Research

mansans

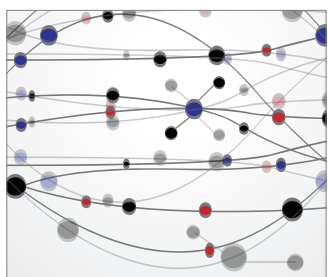

The Scientific World Journal
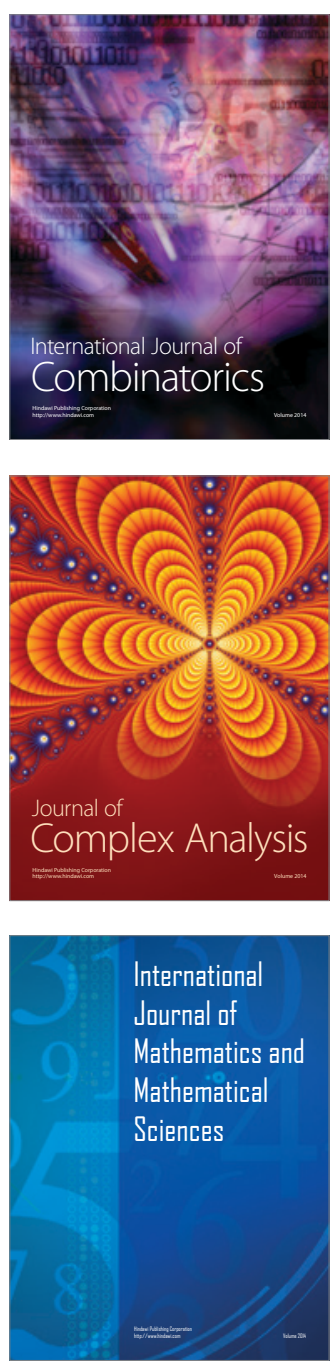
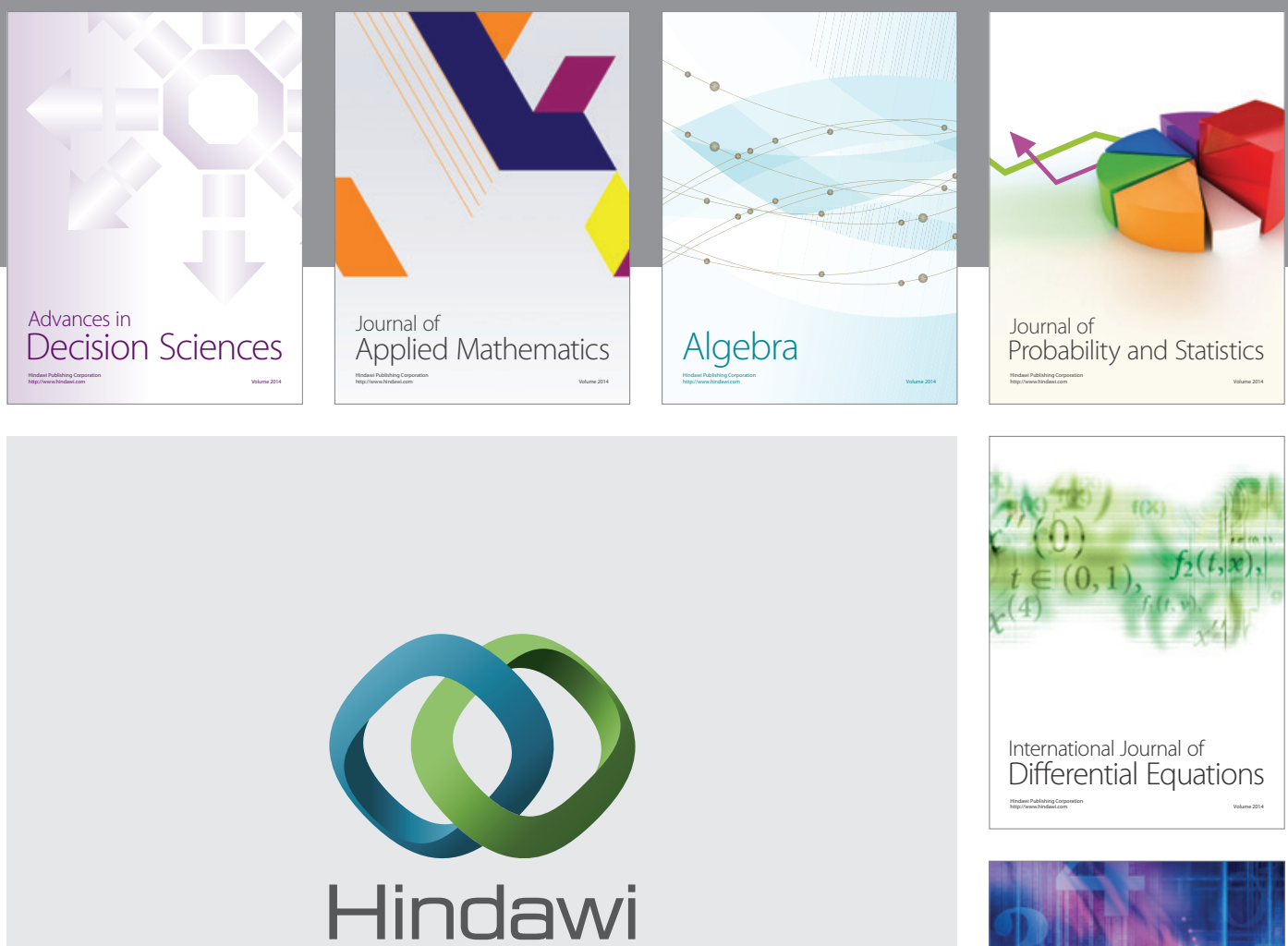

Submit your manuscripts at http://www.hindawi.com
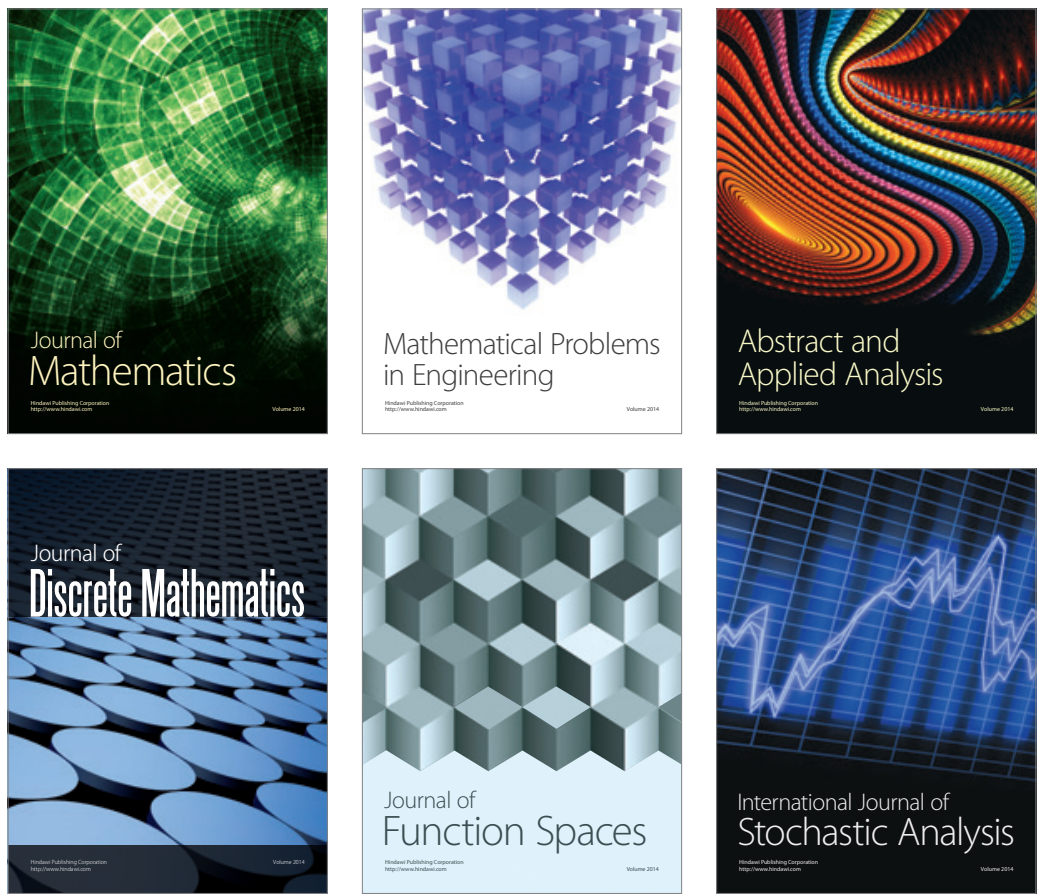

Journal of

Function Spaces

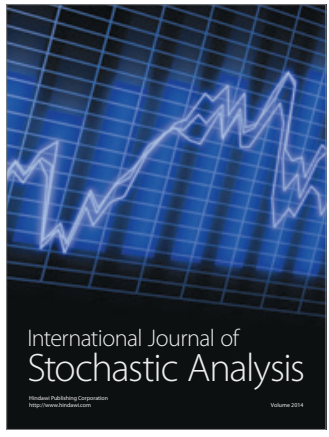

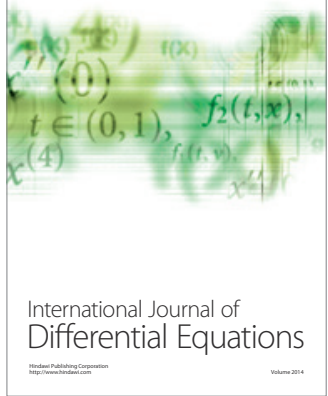
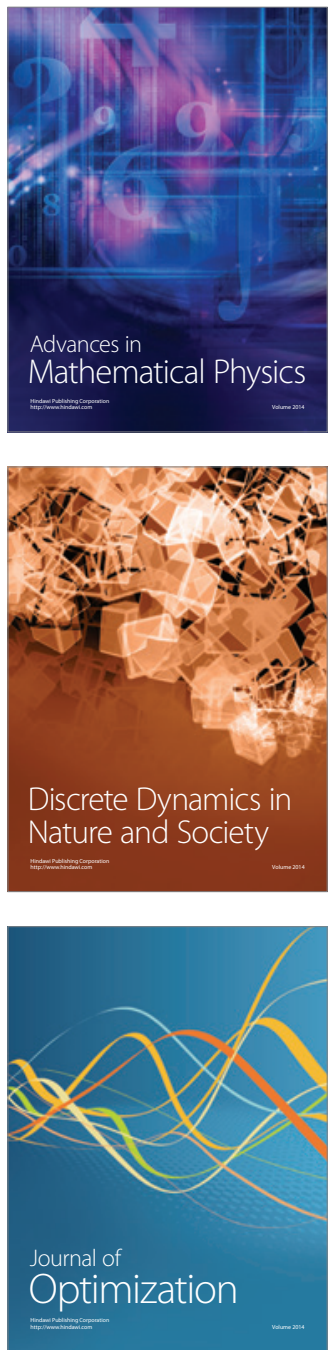